\title{
Unusual Survival of Anomalous Left Coronary Artery From the Pulmonary Artery With Severe Rheumatic Mitral Stenosis in Septuagenarian Women: Foes Becoming Friends?
}

\author{
Santosh Kumar Sinha ${ }^{\mathrm{a}, \mathrm{b}}$, Dibbendhu Khanra ${ }^{\mathrm{a}}$, Mukesh Jitendra Jha ${ }^{\mathrm{a}}$, Karandeep Singh ${ }^{\mathrm{a}}$, Mahamdulla Razi ${ }^{\mathrm{a}}$, \\ Amit Goela, Vikas Mishra a, Mohammad Asifa, Mohit Sachan ${ }^{\mathrm{a}}$, Nasar Afdaali ${ }^{\mathrm{a}}$, Ashutosh Kumara, \\ Ramesh Thakur ${ }^{\mathrm{a}}$, Vinay Krishna ${ }^{\mathrm{a}}$, Umeshwar Pandey ${ }^{\mathrm{a}}$, Chandra Mohan Varma ${ }^{\mathrm{a}}$
}

\begin{abstract}
ALCAPA syndrome (anomalous origin of the left coronary artery from the pulmonary artery) is a rare disease but lethal with clinical expression from myocardial infarction, congestive heart failure to death during early infancy and unusual survival to adulthood. We report a 73-year-old woman with ALCAPA who presented with exertional dyspnea (NYHA functional class II) over past 2 years. Physical examination revealed soft $\mathrm{S}$, long mid diastolic rumbling murmur and apical pan-systolic murmur. Electrocardiography displayed biatrial enlargement and poor R progression and normal sinus rhythm. Echocardiography established calcified severe mitral stenosis (MS), presence of continuous flow entering the pulmonary trunk, turbulent continuous flow in inter-ventricular septum with left to right shunt in contrast echocardiography and normal systolic function. Coronary angiogram showed absence of left coronary artery (LCA) originating from aorta, dilated and tortuous right coronary artery (RCA) and abundant Rentrop grade 3 intercoronary collateral communicating with LCA originating from pulmonary trunk which was also confirmed on coronary CT angiogram thus establishing diagnosis of ALCAPA. It is exceedingly rare to be associated with severe MS. However, such a long survival in our patient can be explained by the severe pulmonary arterial hypertension which may be contributing to lesser coronary steal.
\end{abstract}

Keywords: ALCAPA syndrome; Coronary CT; Contrast echocardiography; Mitral stenosis; NYHA functional class; Rentrop collateral

\section{Introduction}

Anomalous left coronary artery from the pulmonary artery

\section{Manuscript accepted for publication August 08, 2016}

aDepartment of Cardiology, LPS Institute of Cardiology, G.S.V.M. Medical College, Kanpur 208002, Uttar Pradesh, India

${ }^{b}$ Corresponding Author: Santosh Kumar Sinha, Department of Cardiology, LPS Institute of Cardiology, G.S.V.M. Medical College, Kanpur 208002, Uttar Pradesh, India. Email: fionasan@rediffmail.com

doi: http://dx.doi.org/10.14740/jocmr2674w
(ALCAPA) is a rare congenital cardiovascular anomaly occurring only in 1 out of 300,000 live births [1]. Because of profound disturbance of the normal physiology of the myocardium, survival to adulthood is rare [2,3]. Patients with ALCAPA syndrome who survive beyond childhood often have symptoms varying from myocardial ischemia, syncope, arrhythmias to severe ischemic cardiomyopathy which off course will depend on the development of collateral circulation between right coronary artery (RCA) and left coronary system and up to $90 \%$ succumbs by third decade [3]. Survival beyond fourth or fifth decade with ALCAPA syndrome without surgical intervention is exceedingly rare. Sudden deaths occur in about $80-90 \%$ of the infants in absence of appropriate therapy as low pulmonary artery pressure results in coronary steal phenomenon, so survival beyond eighth decade is something very unusual. However, secondary pulmonary arterial hypertension (PAH) because of mitral stenosis (MS) and extensive collateral circulation may delay if not prevent ischemic damage as our patient had no past history of ischemic symptom.

\section{Case Report}

A 73-year-old woman, non-diabetic and non-hypertensive, presented with progressive exertional dyspnea NYHA class III for last 2 years with two episodes of orthopnea in recent past. Blood pressure was $100 / 76 \mathrm{~mm} \mathrm{Hg}$ in right arm in supine position. Pulse rate was $70 / \mathrm{min}$, regular, low volume, with no special character with all peripheral pulses palpable. Jugular venous pulse pressure was elevated $6 \mathrm{~cm}$ above sternal angle with prominent a wave and v-y descent. Apex beat was located in right fifth intercostals space, tapping in character. There was grade II right parasternal heave. P2 was palpable. $\mathrm{S}_{1}$ was soft, $\mathrm{S}_{2}$ was loud; loud $\mathrm{P}_{2}$ component with narrow split. Opening snap (OS) was present with narrow $\mathrm{A}_{2}$-OS gap. There was long grade III mid diastolic rumbling murmur with presystolic accentuation and another soft localized apical pansystolic murmur (Levine grade II/VI) heard at fourth intercostals space lateral to left sterna border with louder diastolic component. Electrocardiogram displayed normal sinus rhythm, biatrial enlargement, left axis deviation, poor R progression and ST-T changes leads $\mathrm{V}_{5-6}$ (Fig. 1). Chest skiagram PA view displayed full pulmonary bay and dilated right pulmonary artery along with features of pulmonary hypertension (Fig. 2). Trans-thoracic echocar- 


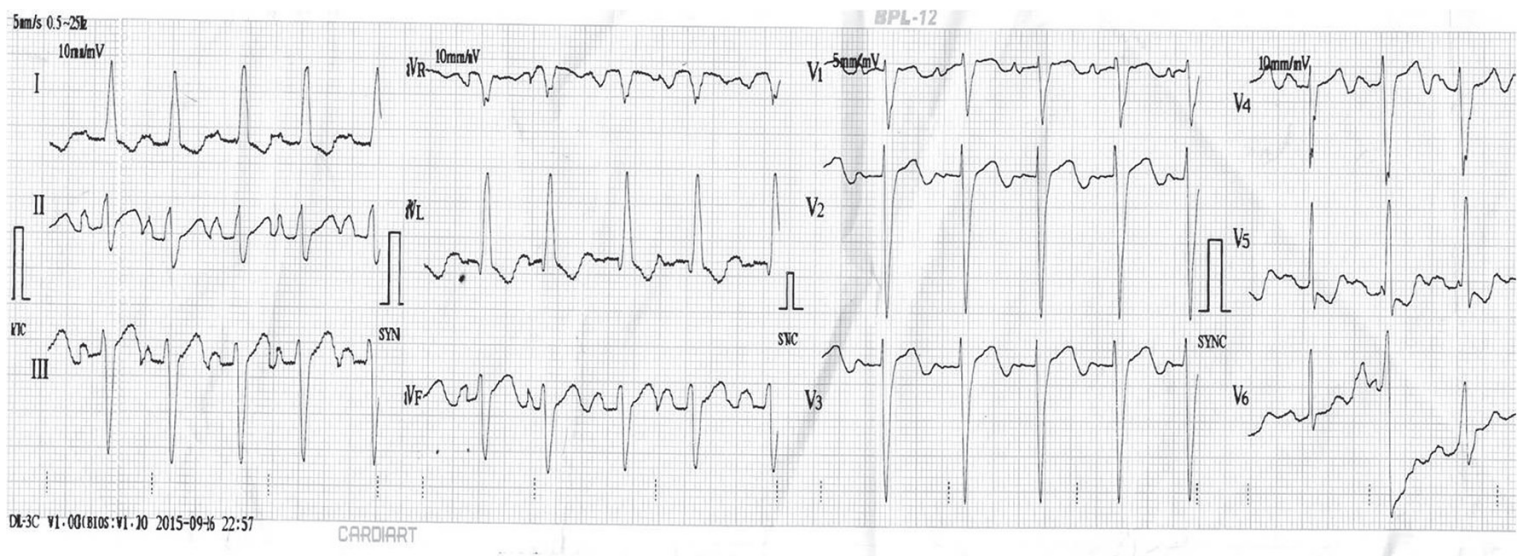

Figure 1. ECG displaying normal sinus rhythm, biatrial enlargement, left axis deviation, poor R progression and ST-T changes in leads $V_{5-6}$.

diogram and color Doppler showed thickened anterior mitral leaflet (AML) and posterior mitral leaflet (PML) with fixed, doming PML confirming its rheumatic etiology. There was severe MS with mitral valve area of $1.2 \mathrm{~cm}^{2}$ by pressure half time and planimetry, mild mitral regurgitation with Wilkin's score of 9/16 $\left(\mathrm{C}_{3}, \mathrm{~T}_{2}, \mathrm{M}_{2}, \mathrm{~S}_{2}\right)$, mean gradient across mitral valve being $18 \mathrm{~mm} \mathrm{Hg}$, and severe tricuspid regurgitation with estimated pulmonary arterial systolic pressure of $77 \mathrm{~mm} \mathrm{Hg}$ (Fig. 3A, B). There was continous flow entering the pulmonary trunk and normal systolic function (Fig. 4). Color Doppler study revealed turbulent continuous flow in interventricular septum with septal fluttering (Fig. 3C, Supplementary video 1, www.jocmr. org). Furthermore, origin of RCA was dilated and LCA was originated from main pulmonary artery (Fig. 3D) with coronary flow seen in medial wall of pulmonary artery near pulmonary valve suggestive of ALCAPA. Complete hemogram, liver, kidney function tests, and serum electrolytes were within normal limit. Because of mild osteoarthritis of both knees, tread mill test could not be done. After proper consent, patient was taken to catheterization lab. Coronary cine-angiogram showed dilated and tortuous RCA (Fig. 5B) with absence of a left coronary

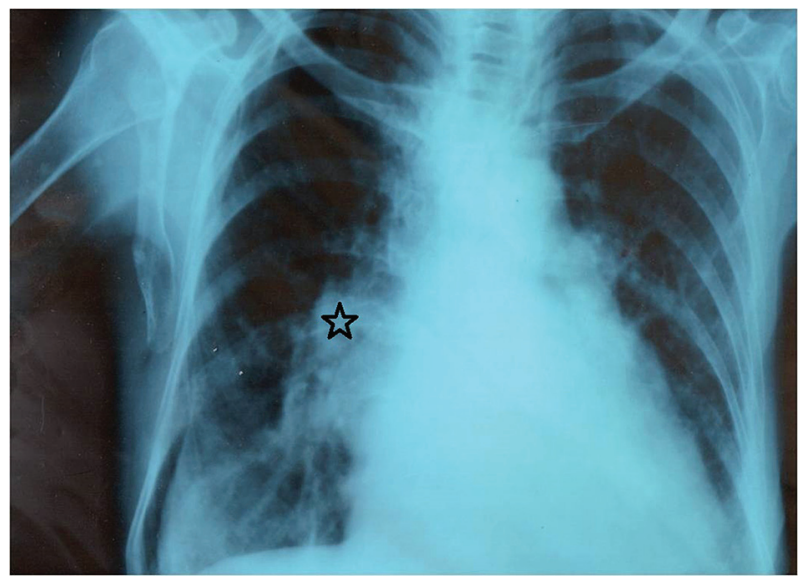

Figure 2. Chest skiagram PA view displaying full pulmonary bay and dilated right pulmonary artery along with features of pulmonary congestion. ostium in the left aortic sinus (Fig. 5A).

During the delayed phase, abundant intercoronary anastomoses (Rentrop grade 3 intercoronary collateral) were seen, communicating with LCA (Fig. 5C) and still very delayed in filming sequence, retrograde flow from the left anterior descending and left circumflex coronary arteries was opacifying the left main coronary artery and its origin from the main pulmonary artery thus establishing diagnosis of ALCAPA. Similar finding was recognized on reconstructed 3D coronary CT angiogram (Fig. 5D). Though surgical treatment by recreating a dual coronary perfusion is usually warranted regardless of the symptoms or myocardial function for ALCAPA and closed mitral valvulotomy for calcific MS, it was decided not to intervene surgically as risk of cardiac surgery was outweighing the benefit and the patient was not willing for surgery and thus she was discharged in stable condition. Till her last follow-up which was 6 months after her discharge, the patient was symptom free and was taking diuretics and beta-blocker.

\section{Discussion}

The estimated incidence of ALCAPA syndrome, a cardiological conundrum is between $0.24 \%$ and $0.46 \%$ of all congenital cardiac anomalies [4]. Most of the patients with ALCAPA generally present within first few months as blood flow through LCA decreases due to gradual reduction of pulmonary arterial pressure in postnatal period. Scarcely some subjects remain asymptomatic until adulthood due to superdominant RCA, although they generally become symptomatic in early adulthood due to fixed myocardial ischemia and coronary steal phenomenon. Wesselhoft et al [1] have proposed the clinical spectrum of ALCAPA spanning from infantile syndrome (most common form, presenting age first few months), mitral regurgitation (presenting age adolescence), syndrome of continuous murmur (due to excess flow between RCA and LCA, presenting at an age of early adulthood), and sudden death in adulthood. Most of the adults are asymptomatic, but some may experience angina on exertion, exertional dyspnea, cardiac arrhythmias and sudden death. Patient's survival without surgical interven- 


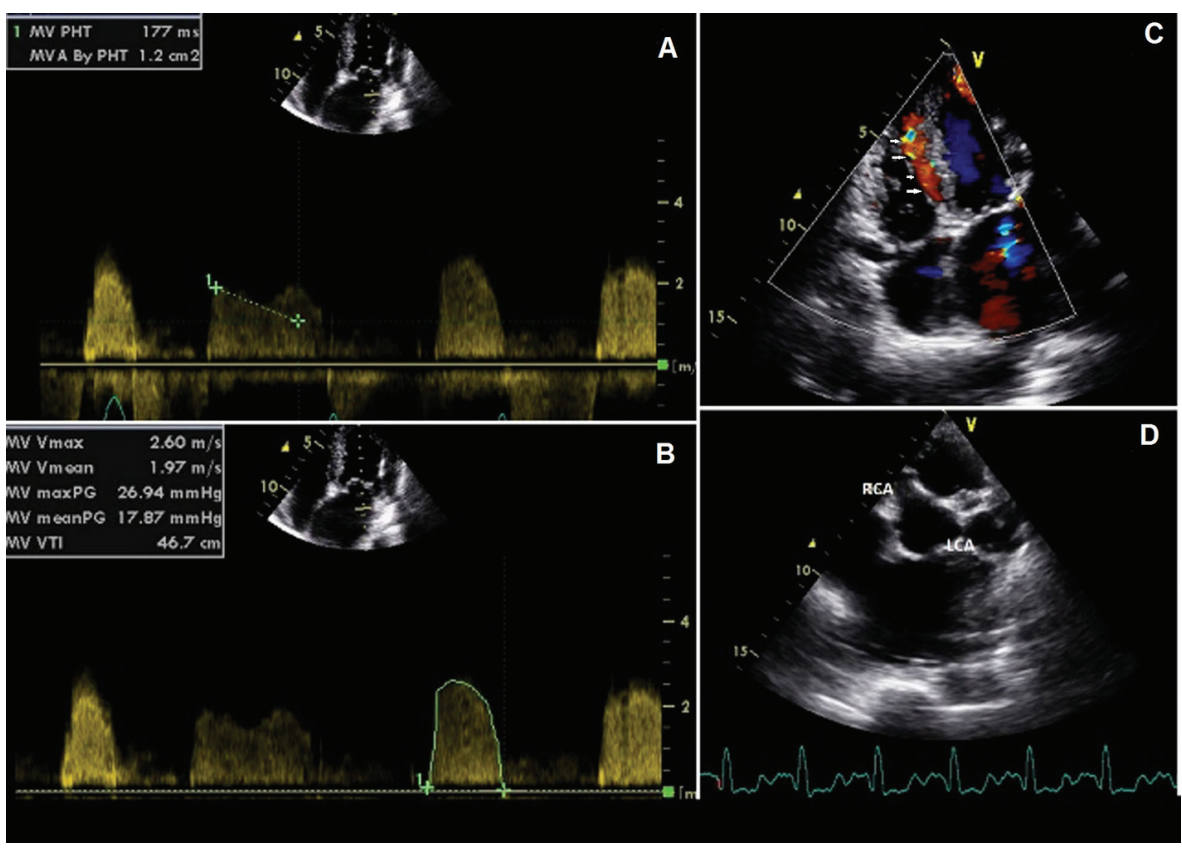

Figure 3. Trans-thoracic echocardiogram and color Doppler showing severe mitral stenosis $(\mathrm{A}, \mathrm{B})$, mild mitral regurgitation and interventricular septal fluttering (white arrow, C), and dilated right coronary artery and left coronary artery originating from main pulmonary artery (D).

tion depends on development of unusually abundant collateral channels from the RCA and closure of the ductus arteriosus. ALCAPA develops before birth and as the systemic and pulmonary arterial pressures are equal, there is anterograde flow in both the left and the right coronary. In the neonatal period as the pulmonary artery pressure falls and ductus arteriosus closes, the flow in the LCA reverses. Trans-thoracic echocardiography is investigation of choice in infants where it demonstrates RCA filling left heart system and retrograde filling of LCA ultimately draining into pulmonary artery. In case of adults coronary artery is hard to delineate by echocardiography. In case of strong suspicion patient should undergo coronary angiography and contrast CT to confirm diagnosis. In our case we followed the same path to diagnosis.

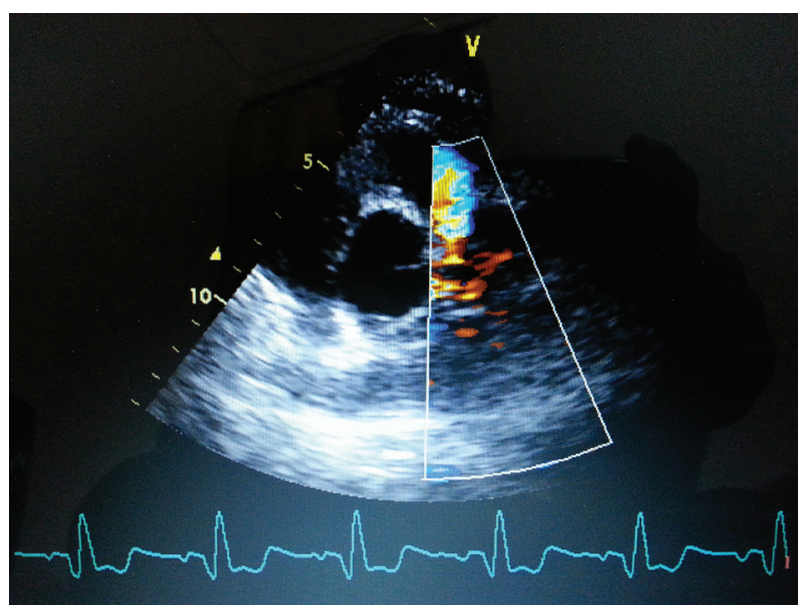

Figure 4. Continuous flow entering the pulmonary trunk.
The more collateral channels exist between RCA and left coronary system, the longer the patient may survive. Another contributing factor may be the area supplied by the ALCA. The smaller the area supplied by the ALCA, the less extensive the myocardial ischemia is likely to be. In few adult cases, RCA supplies not only the posterior wall of the left ventricle but also its lateral wall. May be this reduction in the area of the myocardium supplied by the ALCA would favor survival [1]. However, even extensive collateral circulation may not totally prevent ischemic damage though can delay it as low pulmonary artery pressure may still result in coronary steal phenomenon, which may cause sudden deaths. Nearly $80-90 \%$ of the infants succumb in absence of appropriate therapy. Survival past fourth or fifth decade is rare and untreated ALCAPA in the elderly is exceedingly rare [5] though survival in eighth decade is reported [6].

But ALCAPA has never been found to be associated with rheumatic MS in literature and this is the first case ever to come across. PAH developing due to pulmonary venous hypertension caused by rheumatic MS might have helped this patient to stay relatively asymptomatic despite persistent ALCAPA. High pressure in pulmonary artery may reduce coronary steal phenomenon reducing left-sided ischemia. We believe RCA may have "supernormal" flow reserve to account for her survival.

Surgical correction is treatment of choice in ALCAPA since it is listed as one of the coronary anomalies not compatible to life such as direct transfer of the LCA from pulmonary artery (like in arterial switch operation) [5], Takeuchi repair (creating aorto-pulmonary window) [5], and bypass graft when direct transfer or the Takeuchi repair is not feasible. In our case, as risk of cardiac surgery was outweighing the benefit 


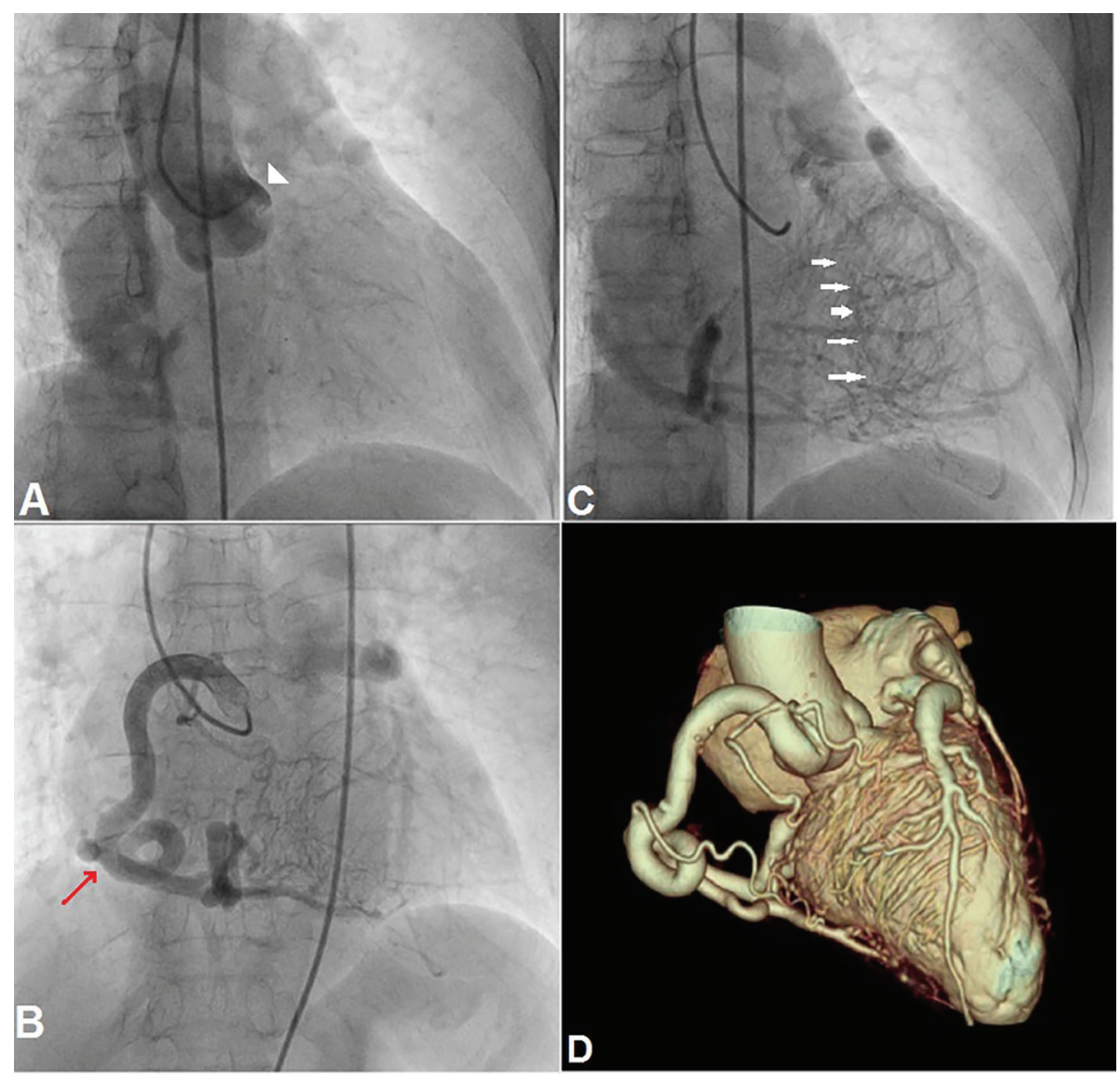

Figure 5. Coronary angiogram showing absence of a left coronary ostium in aortic sinus (white arrow head; A), dilated and tortuous right coronary artery (red arrow; B), intercoronary collaterals communicating with left coronary artery (white arrows; C), and origin of left anterior descending and left circumflex coronary arteries from the main pulmonary artery on reconstructed coronary CT angiogram (D).

and the patient was not willing for surgery, thus she was discharged in stable condition.

\section{Conflicts of Interest}

None.

\section{References}

1. Wesselhoeft H, Fawcett JS, Johnson AL. Anomalous origin of the left coronary artery from the pulmonary trunk. Its clinical spectrum, pathology, and pathophysiology, based on a review of 140 cases with seven further cases. Circulation. 1968;38(2):403-425.

2. Bland EF, White PD, Garland J. Congenital anomalies of the coronary arteries: report of an unusual case associated with cardiac hypertrophy. Am Heart J. 1933;8:787-801.

3. Wilson CL, Dlabal PW, Holeyfield RW, Akins CW, Knauf DG. Anomalous origin of left coronary artery from pulmonary artery. Case report and review of literature concerning teen-agers and adults. J Thorac Cardiovasc Surg. 1977;73(6):887-893.

4. Wollenek G, Domanig E, Salzer-Muhar U, Havel M, Wimmer M, Wolner E. Anomalous origin of the left coronary artery: a review of surgical management in 13 patients. J Cardiovasc Surg (Torino). 1993;34(5):399-405.

5. Alexi-Meskishvili V, Berger F, Weng Y, Lange PE, Hetzer $\mathrm{R}$. Anomalous origin of the left coronary artery from the pulmonary artery in adults. J Card Surg. 1995;10(4 Pt 1):309-315.

6. Santosh Kumar Sinha, Chandra Mohan Varma, Vinay Krishna, Ramesh Thakur, et al. ALCAPA in an Octogenarian Woman: An Enigma. Cardiol Res. 2015;6(3):289291C. 